\title{
Stepwise anti-inflammatory and anti-SARS-CoV-2 effects following convalescent plasma therapy with full clinical recovery
}

\section{Aurelia Zimmerli}

Lausanne University Hospital and University of Lausanne, Switzerland Matteo Monti

Lausanne University Hospital and University of Lausanne, Switzerland

\section{Craig Fenwick}

Lausanne University Hospital and University of Lausanne, Switzerland

\section{Isabella Eckerle}

Geneva University Hospitals, Geneva

\section{Catherine Beigelman-Aubry}

Lausanne University Hospital and University of Lausanne, Switzerland

\section{Celine Pellaton}

Lausanne University Hospital and University of Lausanne, Switzerland

Katia Jaton

Lausanne University Hospital and University of Lausanne, Switzerland

\section{Dominique Dumas}

Lausanne University Hospital and University of Lausanne, Switzerland

\section{Gian-Marco Stamm}

Lausanne University Hospital and University of Lausanne, Switzerland

\section{Laura Infanti}

Regional Blood Transfusion Service, Basel, Switzerland

Heidrun Andreu-Ullrich

Interregional Blood Transfusion SRC, Epalinges, Switzerland

\section{Daphne Germann}

Lausanne University Hospital and University of Lausanne, Switzerland

\section{Marie Mean}

Lausanne University Hospital and University of Lausanne, Switzerland

\section{Peter Vollenweider}

Lausanne University Hospital and University of Lausanne, Switzerland

\section{Raphael Stadelmann}

Lausanne University Hospital and University of Lausanne, Switzerland

\section{Maura Prella}


Lausanne University Hospital and University of Lausanne, Switzerland

\section{Denis Comte}

Lausanne University Hospital and University of Lausanne, Switzerland

\section{Benoit Guery}

Lausanne University Hospital and University of Lausanne, Switzerland

\section{David Gachoud}

Lausanne University Hospital and University of Lausanne, Switzerland

\section{Nathalie Rufer ( $\sim$ Nathalie.Rufer@unil.ch )}

Lausanne University Hospital and University of Lausanne, Switzerland

\section{Case Report}

Keywords: convalescent plasma transfusion, chronic COVID-19 disease, anti-SARS-CoV-2 neutralizing antibodies, anti-inflammatory effect, viral clearance

Posted Date: September 16th, 2020

DOl: https://doi.org/10.21203/rs.3.rs-76799/v1

License: (9) This work is licensed under a Creative Commons Attribution 4.0 International License. Read Full License

Version of Record: A version of this preprint was published at Frontiers in Immunology on September 16th, 2020. See the published version at https://doi.org/10.3389/fimmu.2021.613502. 


\section{Abstract}

Convalescent plasma to treat coronavirus disease 2019 (COVID-19) is currently the focus of numerous clinical trials worldwide, but the criteria of treatment efficacy remain largely unknown. Here, we describe a severely immunosuppressed patient following rituximab and chemotherapy for chronic lymphoid leukemia, who became infected by SARS-CoV-2. His prolonged viral disease was successfully cured after four cycles of convalescent plasma. Its immunomodulatory properties led to the rapid improvement of inflammation, pneumonia and blood cell counts, already after the first cycle. Importantly, the cumulative increase in anti-SARS-CoV-2 neutralizing antibodies following each plasma transfusion was associated to progressive viral clearance, resulting in clinical recovery from infection. Our data provide insight into the different modes of action of plasma components. Understanding the underlying mechanisms will help to optimize the treatment of COVID-19 patients.

\section{Introduction}

COVID-19 is a rapidly evolving novel infectious disease caused by SARS-CoV-2 (severe acute respiratory syndrome coronavirus 2), leading to substantial morbidity and mortality. Due to its worldwide spread, concern has been raised about the potential effect of SARS-CoV-2 infection on immunocompromised patients, particularly for those receiving B-cell depleting agents (e.g. rituximab). While immunosuppressed patients with chronic inflammatory diseases (i.e. multiple sclerosis) do not seem to have an increased risk for a severe COVID-19 course ${ }^{1,2}$, this may not be the case for treated patients with hematological malignancies ${ }^{3}$. Given the lack of effective antiviral therapies or of vaccines available for patients with SARS-CoV-2 infection, plasma provided by convalescent donors (i.e. convalescent plasma) has been proposed as a therapeutic option for COVID-194,5, based on its partial effectiveness in other respiratory infections ${ }^{6,7}$. Consistent with this, recent data have shown a benefit of convalescent plasma in COVID-19 patients with severe respiratory disease ${ }^{8-10}$. It is likely that neutralizing antibodies are important for therapeutic effects ${ }^{11}$. However, convalescent plasma may have additional and currently still unknown mechanisms of action ${ }^{12}$.

\section{Methods}

\section{Ethics statement}

This study was reviewed and approved by the Medical Board Committee of the Lausanne University Hospital (Lausanne, Switzerland). Patient A. P. gave written informed consent. Each donor gave written consent for plasma donation, according to regulations of the Swiss Blood Transfusion Services.

\section{Nasopharyngeal swabs analysis}


Nasopharyngeal swabs were processed under airborne precaution. The SARS-CoV-2 RNA was detected by reverse transcription and real-time PCR assays using a fully automated molecular diagnostic platform MDx platform as described in ${ }^{13}$. Two independent primers targeting RNA polymerase (RdRP) and envelope $(E)$ genes were used as recently submitted ${ }^{14}$. All obtained $\mathrm{Ct}$ values were converted to viral loads based on plasmids positive controls, as previously reported in ${ }^{13}$.

\section{Convalescent donor plasma and transfusion}

Convalescent plasma was collected from three male donors (i.e. plasma 1 to 3), eligible for blood donation according to the requirements of the Blood Donation Service, Swiss Red Cross, who had fully recovered for at least 28 days after COVID-19 onset and had high titers of anti-SARS-CoV-2 IgG (anti-S1 protein, 7.02-9.7 S/CO, by ELISA from Eurolmmun, ${ }^{15}$ ). Apheresis was performed using the Aurora ${ }^{\text {TM }}$ (Fresenius Kabi) or the Trima Accel (Terumo BCT) with the collection of $650 \mathrm{ml}$, sufficient for three units. Manufacturing occurred under good manufacturing practice (GMP) conditions, according to the current regulations (Swisstransfusion CRS and Swiss Federal Act on Medical Products). After collection and before splitting into single units, the leukocyte-depleted plasma was treated for pathogen-inactivation using amotosalen and UVA irradiation (INTERCEPT system, Cerus Corporation). The plasma was further separated into three units $\left(200+/-20 \mathrm{ml}\right.$ each) and kept frozen at $-25^{\circ} \mathrm{C}$.

Patient A. P. was eligible for the study and received four cycles of ABO-identical convalescent plasma transfusion (i.e. two plasma units of $200 \mathrm{ml}$, corresponding to $3 \mathrm{ml} / \mathrm{kg} / \mathrm{unit}$, on two consecutive days of each cycle) obtained from three selected donors, at an interval of 10 to 15 days between the transfusion cycles. Each transfusion was administrated over a 45-minute period, without any adverse events.

\section{Luminex anti-SARS-CoV-2 S protein IgG, IgA and IgM binding assay}

Preparation of the Luminex beads coupled with the $S$ protein trimer was performed as described in ${ }^{16}$. $S$ protein-coupled Luminex beads were added to Bio-Plex Pro 96-well flat bottom plates, washed with PBS, before adding $50 \mathrm{al}$ of a 1/300 dilution of individual serum (from the patient) or plasma (from the donors) to the wells ${ }^{16}$. Plates were agitated at $500 \mathrm{rpm}$ for 60 minutes on a plate shaker. Beads were then washed and anti-human IgG-PE or anti-human IgA-PE (OneLambda ThermoFisher) or anti-human IgM secondary antibody (BioConcept) was added. Plates were agitated for 45 minutes and washed again. Beads were resuspended in $80 \mathrm{Ll}$ of reading buffer and read directly on a Luminex FLEXMAP 3D plate reader (ThermoFisher). MFI signal of each test serum or plasma sample was divided by the mean signal for the negative control samples yielding a MFI ratio ${ }^{16}$.

Quantitative analysis of serum or plasma levels of anti-S protein IgG antibodies was determined using MFI signal from $1 / 300,1 / 900,1 / 2700$ and $1 / 81000$ dilutions of sample compared to a standard curve 
generated with a purified anti-SARS-CoV-2 S protein monoclonal antibody (Clone CR3022; Lucerna Chem).

\section{SARS-CoV-2 pseudovirus neutralization assay}

A single-cycle infectivity assay was used to measure the neutralization of luciferase-encoding virions pseudotyped expressing the trimeric form of the S glycoprotein of SARS-CoV-2. Supernatants containing SARS-CoV-2 pseudovirus were preincubated with serially diluted plasma from the three donors or serum from the patient at different time-points before and after plasma transfusions. After $1 \mathrm{~h}$ at $37^{\circ} \mathrm{C}$, all preincubated supernatants were added on ACE-2 transfected $293 \mathrm{~T}$ cells and incubated for $72 \mathrm{~h}$ at $37^{\circ} \mathrm{C}$. The luciferase activity was measured using the EnVision. $\mathrm{IC}_{50}$ (serum dilution) was calculated by nonlinear regression analysis using the GraphPad Prism 8.3 software.

\section{SARS-CoV-2 viral cultures}

For assessment of infectious virus, VeroE6 cells were seeded at a density of $8 \times 10^{4}$ cells/well in a 24 well plate and inoculated with $200 \mu$ l of viral transport medium the following day as reported in ${ }^{17}$. Cells were inoculated for $1 \mathrm{~h}$ at $37^{\circ} \mathrm{C}$, then inoculum was removed and cells were further grown in regular cell medium. Cells were observed on day 3 and 7 or the presence of cytopathic effect (CPE) by light microscopy. In addition, supernatant was harvested immediately after inoculation and at each follow-up time-point. Isolation of replication competent SARS-CoV-2 was confirmed by presence of a CPE and an increase in viral RNA between consecutive time-points ${ }^{17}$.

\section{Safety and therapeutic outcome evaluation}

The clinical symptoms were recorded by attending nurses and physicians daily. Following convalescent plasma transfusion, baseline laboratory exams (renal and hepatic function, complete blood count), biochemical (CRP and ferritin), lymphocyte enumeration (CD4, CD8 and B cell count) and antibody titers (anti-SARS-CoV-2 IgG, IgA and IgM) as well as anti-SARS-CoV-2 neutralizing antibody activity were regularly evaluated, while SARS-CoV-2 RNA was controlled by nasopharyngeal swabs every 2-3 days. Surveillance of plasma transfusion related adverse events and all severe adverse events was performed until day 7 after each transfusion. Chest CT scan was performed regularly before and after plasma transfusion as following; day 1 , day 27 , day 44 , day 71 , day 80 (post $1^{\text {st }}$ cycle of plasma transfusion) and day 101 (post $3^{\text {rd }}$ cycle of plasma transfusion). The primary outcome was the improvement in symptoms, biological parameters and chest CT scan lesions. The secondary outcome was the evaluation of isolation measures.

\section{Results And Discussion}


Here, we present the case of a 74-year-old man in complete remission of a chronic lymphoid leukemia (CLL) after 6 cycles of rituximab and bendamustin (last therapy administrated in December 2019). On April $1^{\text {st }}$, he presented to our emergency unit with asthenia, loss of weight, dry cough and diarrhea since a month. He was otherwise healthy with well-controlled arterial hypertension and type 2 diabetes mellitus. SARS-CoV-2 RNA was detected $\left(7 \times 10^{6}\right.$ copies $\left./ \mathrm{ml}\right)$ from a nasopharyngeal swab (defined as day 1$)$. The patient presented a moderate neutropenia, but severe $T\left(114 \mathrm{cell} / \mathrm{mm}^{3}\right)$ and $B\left(1 \mathrm{cell} / \mathrm{mm}^{3}\right)$ lymphopenia with reduced total immunoglobulin (Ig) G and IgM levels (Supp. Fig. 1), while inflammatory markers (Creactive protein and ferritin) were elevated (Fig. 1A). Chest computed tomography (CT) revealed bilateral multifocal subpleural and peribronchial ground-glass opacities typical of COVID-19 pneumonia ${ }^{18}$ (Fig. 1B, Supp. Fig. 2). The clinical condition gradually deteriorated with sub-febrile episodes, persisting dry cough and diarrhea, and progressive weight loss and cognitive dysfunction (Fig. 1C, D; Suppl. Information). Inflammatory parameters and blood cell counts remained abnormal (Fig. 1A, E), in line with the repeated SARS-CoV-2 positive nasopharyngeal swabs at high copy numbers (Suppl. Fig. 1). Complementary investigations excluded other diagnoses, while persisting SARS-CoV-2 infection was confirmed (Suppl. Information, Suppl. Fig. 1). No specific antiviral agents were introduced given the mild symptoms of COVID-19 (e.g. absence of hypoxemia).

In summary, the patient presented a long-lasting SARS-CoV-2 infection likely related to his severe immunosuppressive status. Consequently, we hypothesized that convalescent plasma could be beneficial in this particular case, by providing virus-specific neutralizing antibodies as well as a potential antiinflammatory effect. The first cycle of ABO-compatible plasma transfusion (two units on two consecutive days) was given on days 72 and 73 after diagnosis of SARS-CoV-2 infection, followed by three additional cycles, administered 10 to 15 days apart (Suppl. Fig. 3). Plasma units from three convalescent donors were selected, each with relatively high IgG antibody titers against the S1 (spike)-protein using ELISA (Supp. Fig. 3). Within the first eight days after the start of plasma transfusions, the patient improved clinically, biologically and radiologically (Fig. 1). We observed a rapid normalization of the Creactive protein, while absolute platelet counts and hemoglobin levels showed a more gradual return-tonormal. Follow-up chest CT scan confirmed a significant improvement in pulmonary infiltrates (Fig. 1B, Supp. Fig. 2). Absolute lymphocyte counts also substantially improved, and largely consisted of increased levels of memory-effector CD4 and CD8 T cells and of NK cells (Fig. 2A, Supp. Fig. 4). Only a moderate rise was observed for total B cell counts (mostly unswitched memory B cells at day 121), while total IgM and IgG remained globally stable (Fig. 2B, Supp. Fig. 1 and 4). These data indicate clear improvements of inflammation, pneumonia and blood cell counts, already after the $1^{\text {st }}$ cycle of convalescent plasma transfusion.

Most patients with COVID-19 develop SARS-CoV-2 IgM and IgG antibody responses within 19 days after symptom onset ${ }^{19}$. To investigate whether such antibodies were transferred to our patient following successive cycles of plasma transfusion, we monitored the anti-SARS-CoV-2 S protein-specific $\lg G, \lg A$ and IgM antibody levels by Luminex. There was a remarkable heterogeneity among the three plasma donors, with plasma/donor 3 exhibiting highest levels of specific antibodies (Fig. 2C, Supp. Fig. 3). 
Whereas no anti-S IgG response was detected in the patient's serum before the start of plasma transfusion, the antibody titers increased progressively, up to 30-fold over the baseline reference after the $4^{\text {th }}$ cycle (Fig. 2D, Supp. Fig. 3). A similar trend, albeit at much lower levels, was found for anti-S IgA antibodies. Instead, anti-S IgM antibodies revealed two peaks following serial plasma transfusions (Fig. 2D), again related to the level of specific-lgM antibodies of each plasma donor, with plasma/donor 3 showing highest titers (Fig. 2C). We also observed a boost of SARS-CoV-2 neutralizing activity after each cycle of plasma administration, in line with the higher antibody activity found in plasma/donor 3 when compared to the two others (Fig. 2E). Importantly, passive transfer of SARS-CoV-2 neutralizing antibodies inversely correlated to the gradual decline observed in viral loads in nasopharyngeal swabs, becoming undetectable for both $E$ and RdRP genes by day 111 of diagnosis (Fig. 2F). To assess whether shedding of infectious SARS-CoV-2 still occurred after the start of plasma transfusions, we measured the presence of cultivable SARS-CoV-2 at different time-points. While infectious virus could be isolated from nasopharyngeal swabs prior and following the $1^{\text {st }}$ cycle of convalescent plasma transfusion, this was no longer the case upon the $2^{\text {nd }}$ cycle (Fig. 2F). Finally, the patient received one dose of intravenous immunoglobulin (IVlg; $0.4 \mathrm{~g} / \mathrm{kg}$ ) on day 114 , providing additional passive immune protection against common pathogens, before being discharged to home. He was then followed by weekly outpatient care and considered as cured after two weeks of consecutive negative swabs (on day 127), but remaining potentially vulnerable to SARS-CoV-2 re-infection.

Collectively, our study offers novel evidence for a clear benefit of convalescent plasma in this particular case of COVID-19 disease, with the resolution of clinical, inflammatory and radiological parameters (Fig. 1). Improvement of inflammation, pneumonia and blood cell counts preceded viral clearance. Importantly, this patient showed a temporal association between detection of SARS-CoV-2 neutralizing antibodies and virus clearance following successive cycles of plasma transfusion (Fig. 2). Thus, our data provide insight into at least two distinct modes of action of plasma components. The first one is related to its proposed anti-inflammatory activity, similar to IVIg, widely used at high-dose for the treatment of several autoimmune diseases. In this line, convalescent plasma therapy may help in modulating the immune response via $\mathrm{F}\left(\mathrm{ab}^{\prime}\right)_{2}$-dependent mechanisms including blockade of cell-cell interactions (via cell-surface receptors) and neutralization of cytokines, complement and autoantibodies (by anti-idiotypic antibodies) ${ }^{12,20}$. In addition, convalescent plasma activity might involve Fc-dependent pathways (e.g. modulation of Fc $\square$ receptors on innate immune effector cells and B cells) ${ }^{12,20}$. However, unlike IVIg requiring the repetitive infusion of large amounts of Ig to achieve anti-inflammatory activity, this effect was readily observed after the $1^{\text {st }}$ cycle of convalescent plasma transfusion. Of note, the total IgG found within convalescent plasma was 10 to 20 -fold less concentrated as compared to IVIg, largely composed of monomeric IgG (Suppl. Fig. 3). Consequently, the immunomodulatory mechanisms by which immune plasma contributes to control COVID-19 pathogenesis may differ from those of the IVIg immunotherapy ${ }^{21}$ and deserves further studies.

Our observations support a second mechanism of action by SARS-CoV-2-specific IgG and neutralizing antibodies present in convalescent immune plasma (Fig. 2), which may mediate direct virus 
neutralization or other antibody-mediated pathways (e.g. complement activation, antibody-dependent cellular cytotoxicity). Passive antibody therapy has the great advantage to confer immediate immunity to vulnerable individuals ${ }^{22}$. Interestingly, our data further suggest a relatively rapid effect of convalescent plasma with disappearance of cultivable SARS-CoV-2 readily after the second cycle of transfusion, despite intermediate levels of neutralizing antibodies and high RNA-positivity by qRT-PCR (Fig. 2). This is in line with recent findings showing that neutralizing antibodies derived from COVID-19 patients may be effective, even at concentrations of $9 \mathrm{ng} / \mathrm{ml}$ or less ${ }^{23}$. Strikingly, successive cycles of plasma transfusion (every 10-15 days) led to the cumulative increase in anti-S IgG titers, associated with a boost of neutralizing antibodies after each administration. Anti-S-specific IgM titers also followed a kinetic pattern related to the antibody levels of each transfused donor-plasma unit, indicating that a de novo endogenous antibody response was improbable at this stage. Finally, it is certainly possible that CD4 and CD8 T cells played critical roles ${ }^{24}$ in the recovery of this patient who was primarily B cell deficient. This view is supported by reported cases of agammaglobulinemia patients who only presented mild COVID19 disease ${ }^{25}$. In summary, convalescent immune plasma therapy revealed stepwise anti-inflammatory and anti-SARS-CoV-2 effects, resulting in full clinical recovery from infection. It remains to be seen how this compares to future monoclonal anti-SARS-CoV-2 antibodies and other novel COVID-19 therapies.

\section{Declarations}

ACKNOWLEDGMENTS: This study was supported in part by the Department of Internal Medicine and the Department of Oncology (Lausanne University Hospital and University of Lausanne), and Interregional Blood Transfusion SRC (Lausanne, Switzerland). We are grateful to patient A. P. and the blood donors for their dedicated collaboration in this study. We thank Drs. V. Aubert, W. Bosshard, M. Benazza, M. Caci Riedweg, E. Glampedakis, E. Jaquet, D. Samim, L. Senn, J. P. Simon, D. Solari, M. Touray, and the participating medical and laboratory teams of the Lausanne University Hospital (Lausanne, Switzerland) as well as the blood center teams of the Interregional Blood Transfusion SRC (Bern-Vaud-Valais, Switzerland) and the Regional Blood Transfusion Service (Basel, Switzerland). We thank Prof. G. Pantaleo for providing the technical platform for the pseudovirus neutralization assay. We are thankful to Dr. M. Essaidi-Laziosi and F. Pigny for their technical help and to Prof. Daniel E. Speiser, Dr. M. Prudent and Dr. K. Vincent for comments and critical reading of the manuscript. Finally, we thank Prof. Gérard Waeber, head of the Department of Medicine, for his advice regarding patient care, as well as for his comments and critical reading of the manuscript.

AUTHOR CONTRIBUTIONS: Study design and supervision: MMo, BG, DGa and NR. Patient care and acquisition of data: $A Z, M M o, C B, D D, G M S, D G e, M M e, P V, R S, M P, D C, B G$ and DGa. Development of methodology: $C F, I E, C P, L I$, and $H A U$. Analysis and interpretation of data: $A Z, C F, I E, C B, C P, K J, D C, D G a$ and NR. Writing of the manuscript: AZ, DGa and NR.

DISCLOSURE OF POTENTIAL CONFLICTS OF INTEREST: The authors report no conflict of interest. 


\section{References}

1. Montero-Escribano, P., et al. Anti-CD20 and COVID-19 in multiple sclerosis and related disorders: A case series of 60 patients from Madrid, Spain. Mult Scler Relat Disord 42, 102185 (2020).

2. Giovannoni, G., et al. The COVID-19 pandemic and the use of MS disease-modifying therapies. Mult Scler Relat Disord 39, 102073 (2020).

3. Tepasse, P.R., et al. Persisting SARS-CoV-2 viraemia after rituximab therapy: two cases with fatal outcome and a review of the literature. Br J Haematol 190, 185-188 (2020).

4. Casadevall, A. \& Pirofski, L.A. The convalescent sera option for containing COVID-19. J Clin Invest 130, 1545-1548 (2020).

5. Chen, L., Xiong, J., Bao, L. \& Shi, Y. Convalescent plasma as a potential therapy for COVID-19. Lancet Infect Dis 20, 398-400 (2020).

6. Tiberghien, P., et al. Collecting and evaluating convalescent plasma for COVID-19 treatment: why and how? Vox Sang (2020).

7. Mair-Jenkins, J., et al. The effectiveness of convalescent plasma and hyperimmune immunoglobulin for the treatment of severe acute respiratory infections of viral etiology: a systematic review and exploratory meta-analysis. J Infect Dis 211, 80-90 (2015).

8. Duan, K., et al. Effectiveness of convalescent plasma therapy in severe COVID-19 patients. Proc Natl Acad Sci U S A 117, 9490-9496 (2020).

9. Shen, C., et al. Treatment of 5 Critically III Patients With COVID-19 With Convalescent Plasma. JAMA (2020).

10. Li, L., et al. Effect of Convalescent Plasma Therapy on Time to Clinical Improvement in Patients With Severe and Life-threatening COVID-19: A Randomized Clinical Trial. JAMA (2020).

11. Abraham, J. Passive antibody therapy in COVID-19. Nat Rev Immunol 20, 401-403 (2020).

12. Rojas, M., et al. Convalescent plasma in Covid-19: Possible mechanisms of action. Autoimmun Rev 19, 102554 (2020).

13. Greub, G., Sahli, R., Brouillet, R. \& Jaton, K. Ten years of R\&D and full automation in molecular diagnosis. Future Microbiol 11, 403-425 (2016).

14. Jacot, D., Greub, G., Jaton, K., \& Opota, O. Viral load of SARS-CoV-2 across patients and compared to other respiratory viruses. medRxiv, https://doi.org/10.1101/2020.07.15.20154518

15. Harvala, H., et al. Convalescent plasma therapy for the treatment of patients with COVID-19: Assessment of methods available for antibody detection and their correlation with neutralizing antibody levels. medRxiv, https://doi.org/10.1101/2020.05.20.20091694

16. Fenwick, C., et al. Changes in SARS-CoV-2 antibody responses impact the estimates of infections in population-based seroprevalence studies. medRxiv, https://doi.org/10.1101/2020.07.14.20153536

17. L'Huillier, A.G., Torriani, G., Pigny, F., Kaiser, L. \& Eckerle, I. Culture-Competent SARS-CoV-2 in Nasopharynx of Symptomatic Neonates, Children, and Adolescents. Emerg Infect Dis 26(2020). 
18. Ye, Z., Zhang, Y., Wang, Y., Huang, Z. \& Song, B. Chest CT manifestations of new coronavirus disease 2019 (COVID-19): a pictorial review. Eur Radio/ 30, 4381-4389 (2020).

19. Long, Q.X., et al. Antibody responses to SARS-CoV-2 in patients with COVID-19. Nat Med 26, 845-848 (2020).

20. Lunemann, J.D., Nimmerjahn, F. \& Dalakas, M.C. Intravenous immunoglobulin in neurology--mode of action and clinical efficacy. Nat Rev Neurol 11, 80-89 (2015).

21. Bonam, S.R., Kaveri, S.V., Sakuntabhai, A., Gilardin, L. \& Bayry, J. Adjunct Immunotherapies for the Management of Severely III COVID-19 Patients. Cell Rep Med 1, 100016 (2020).

22. Bloch, E.M., et al. Deployment of convalescent plasma for the prevention and treatment of COVID-19. J Clin Invest 130, 2757-2765 (2020).

23. Liu, L., et al. Potent neutralizing antibodies directed to multiple epitopes on SARS-CoV-2 spike. Nature (2020).

24. Altmann, D.M. \& Boyton, R.J. SARS-CoV-2 T cell immunity: Specificity, function, durability, and role in protection. Sci Immunol 5(2020).

25. Quinti, I., et al. A possible role for B cells in COVID-19? Lesson from patients with agammaglobulinemia. J Allergy Clin Immunol 146, 211-213 e214 (2020).

\section{Figures}


A.

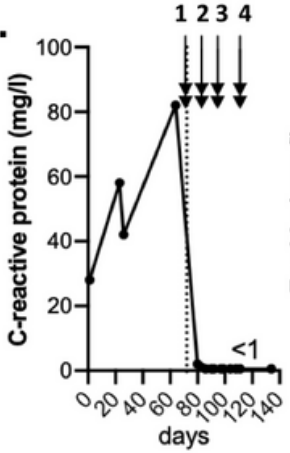

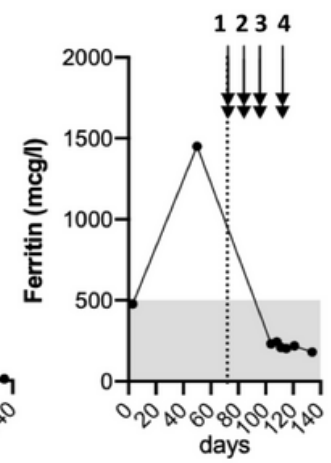

C1 C2 C3 C4

B.

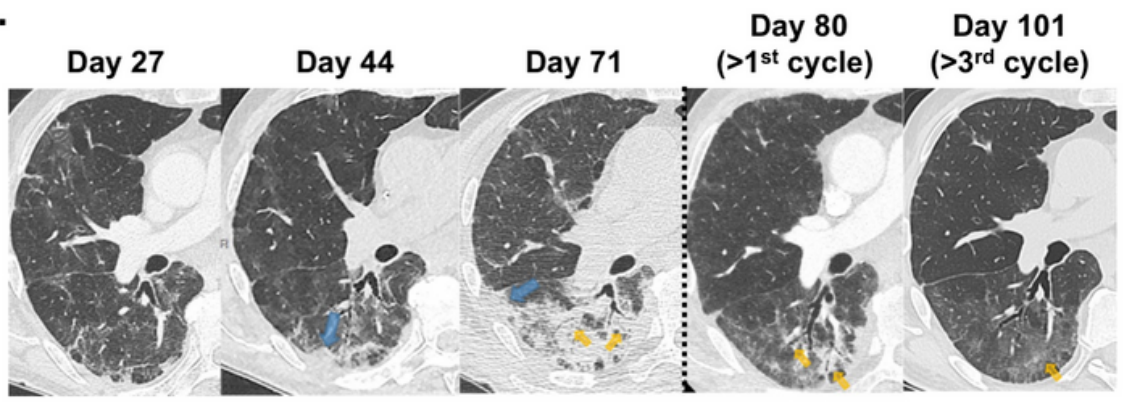

C.
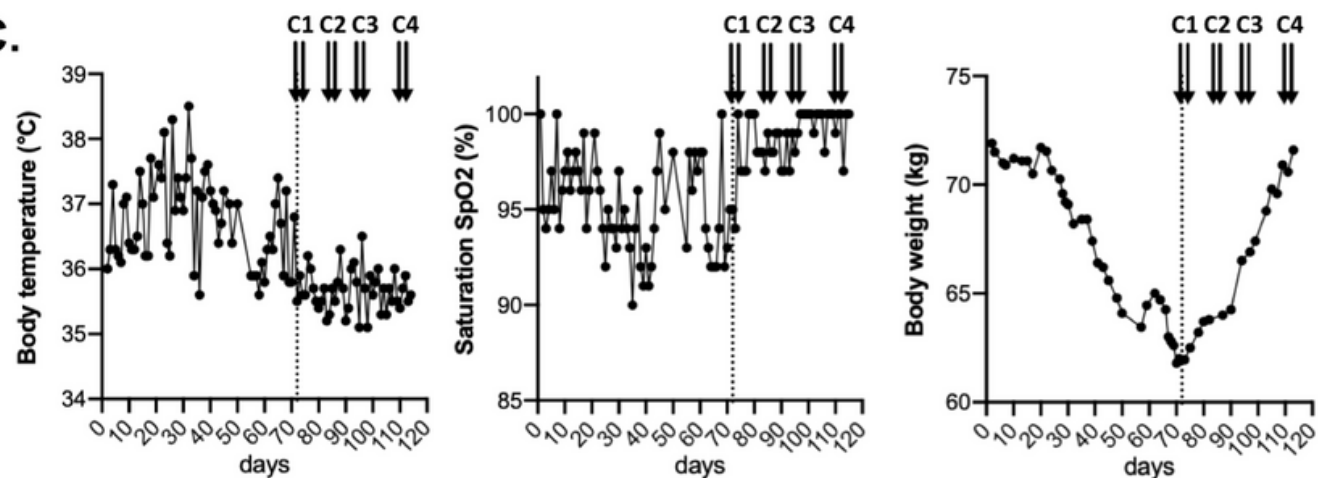

D.

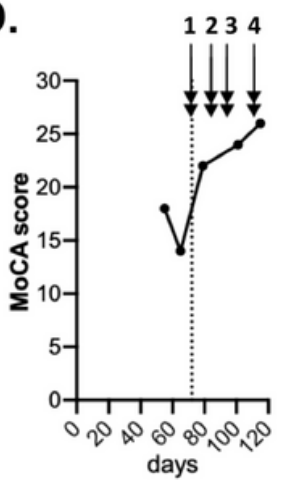

E.
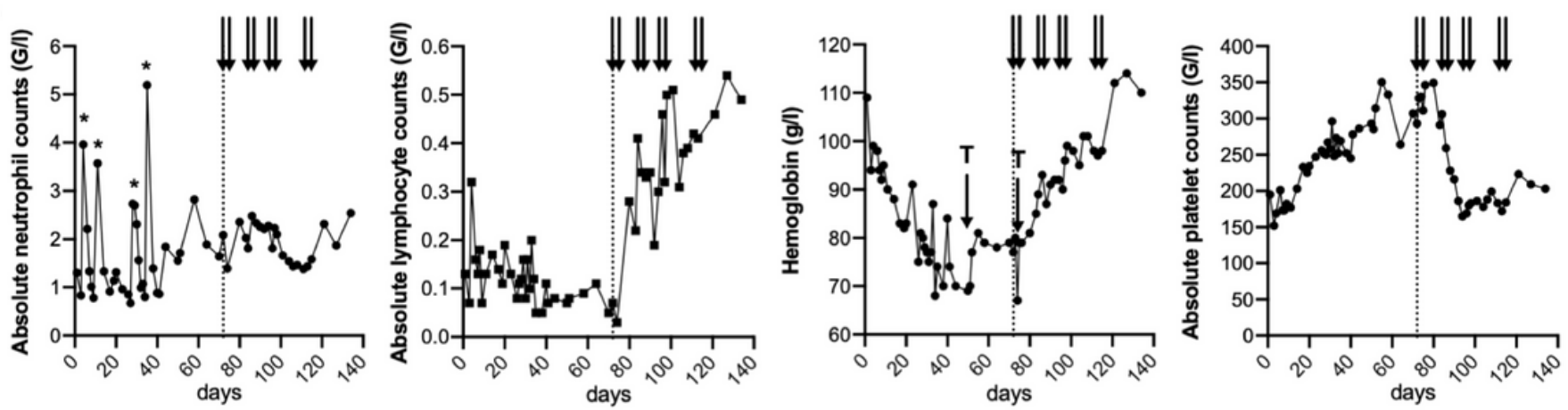

Figure 1

Rapid improvement of clinical, inflammatory and radiological parameters following plasma transfusion. A-E, Timeline of chronic SARS-COV-2 infection in a severely immunosuppressed patient showing inflammatory markers (A), chest CT scan (B), clinical parameters (C), MoCA (Montreal Cognitive Assessment) scores (D), and complete blood counts (E). B, Axial slices focused at the level of the apical segment of the right lower lobe. Progressive worsening from multiple ill-defined areas of ground glass opacities progressing to patchy alveolar consolidation of increasing size. Note the subpleural (blue arrows) and peribronchial (orange arrows) distribution of the lesions with progressive improvement on post-therapeutic follow-up, alveolar consolidation being replaced by ground glass opacity. A-E, Arrows indicate the 4 cycles of plasma transfusion (two units given on two consecutive days of each cycle). The "*" show the 3 injections of granulocyte-colony stimulating factor (filgrastim), while " $T$ " indicates the 2 red blood cell transfusions. 
A.

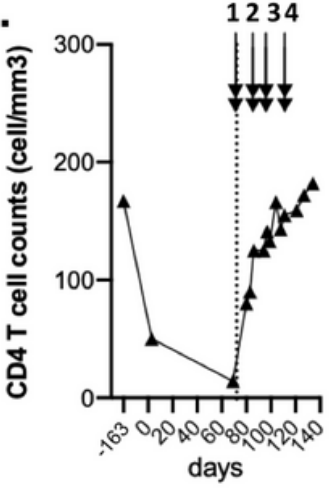

C.

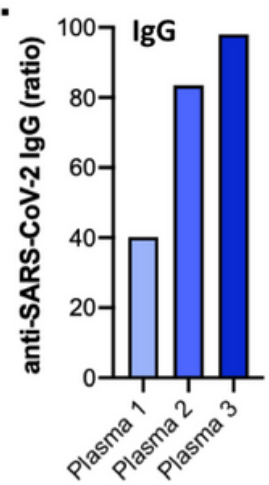

D.

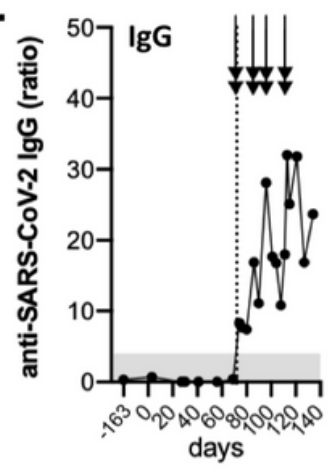

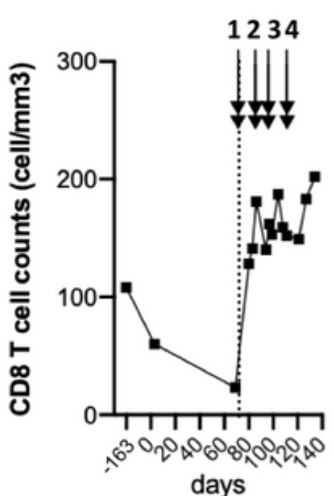
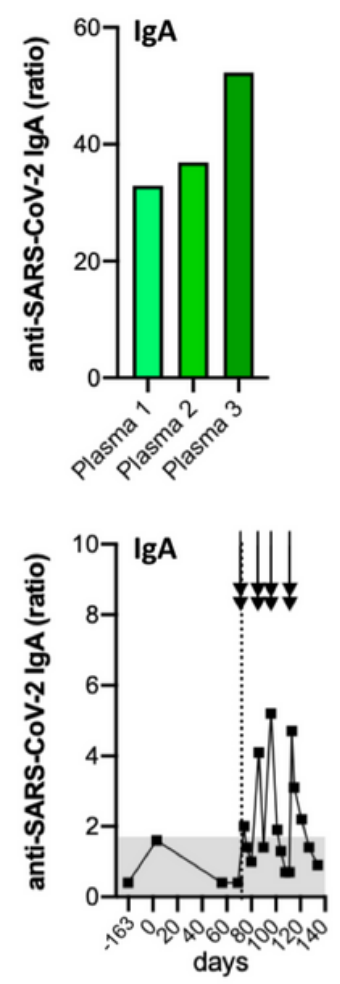
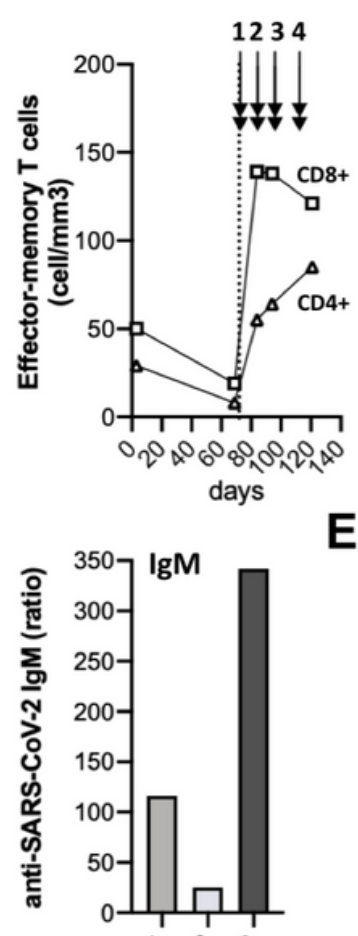

E.
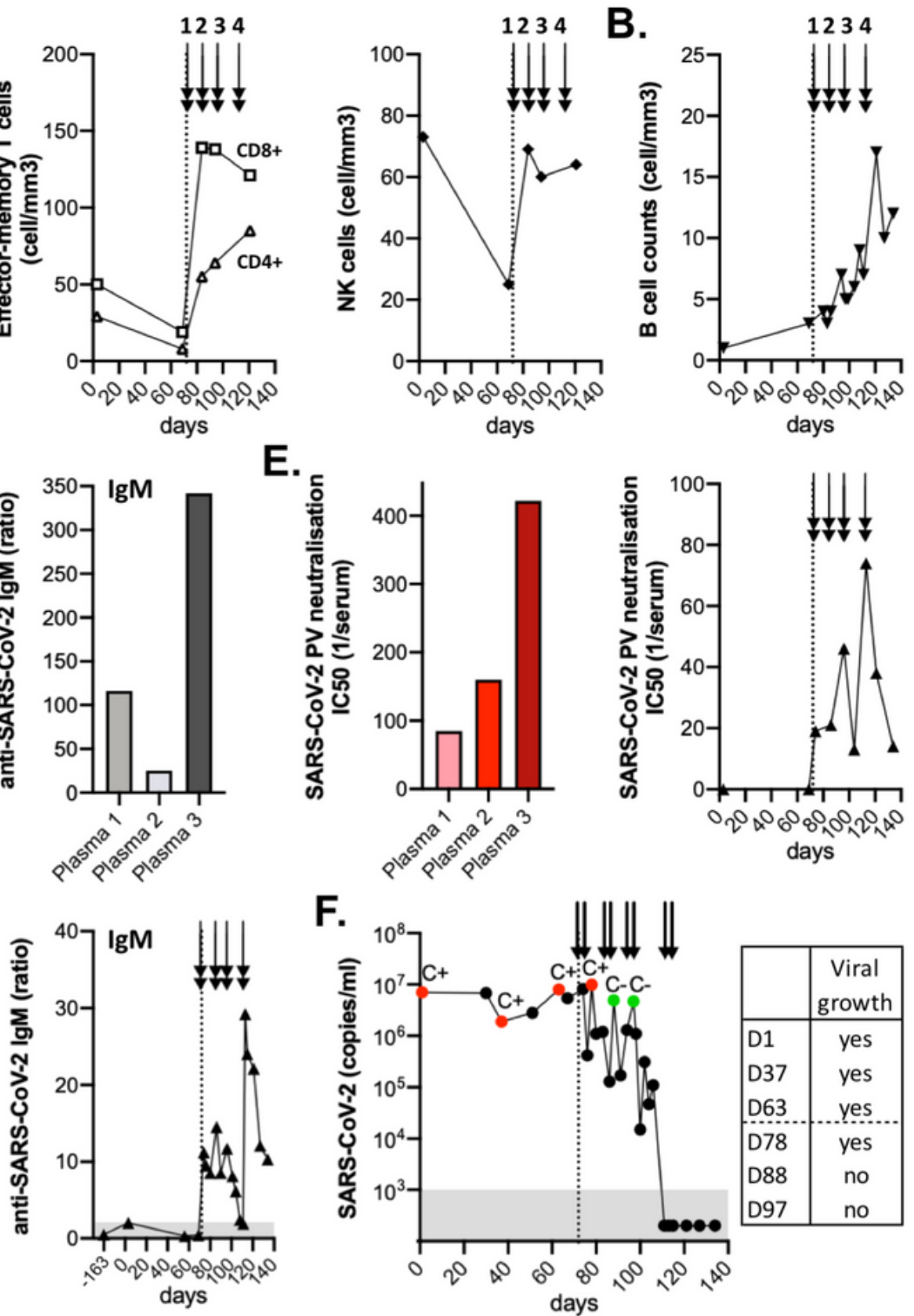

F.

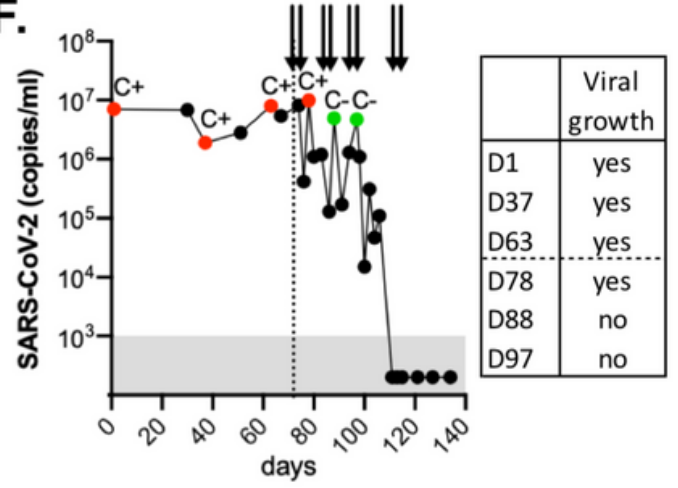

Figure 2

Temporal association between detection of SARS-CoV-2 neutralizing antibodies and viral clearance. A-B, Timeline showing absolute CD4 and CD8 T cell counts, including effector-memory subsets and NK cell counts (A), and absolute B cell counts (B). C and D, Anti-SARS-CoV-2 S protein IgG, IgA and IgM antibody levels as assessed by Luminex for each plasma (C) as well as in the patient's serum before and following plasma transfusions (D). E, Activity of neutralizing antibodies was assessed by a SARS-CoV-2 pseudovirus neutralization assay for each plasma and in patient's serum at different time-points. F, Overtime follow-up of SARS-CoV-2 RNA detection in nasopharyngeal swabs. The cytopathic effect on VeroE6 cells was evaluated after inoculation with SARS-CoV-2 from nasopharyngeal swabs: $\mathrm{C}+$, successful virus isolation; $C$-, absence of virus isolation. A-F, The arrows indicate the 4 cycles of plasma transfusion (two units given on two consecutive days of each cycle). 


\section{Supplementary Files}

This is a list of supplementary files associated with this preprint. Click to download.

- ZimmerlietalSupplementallnfoSuppFigures14.pdf 